tES AND ADHD

\title{
Resolving Heterogeneity in Transcranial Electrical Stimulation Efficacy for Attention Deficit Hyperactivity Disorder
}

Renée Lipka ${ }^{\mathrm{a}}$, Eike Ahlers ${ }^{\mathrm{a}}$, Thomas L. Reed ${ }^{\mathrm{b}}$, Malin I. Karstens ${ }^{\mathrm{b}}$, Vu Nguyen ${ }^{\mathrm{c}}$, Malek Bajbouj $^{\mathrm{a}}$, Roi Cohen Kadosh ${ }^{\mathrm{b}}$

a Charité Universitätsmedizin, Department of Psychiatry, Campus Benjamin Franklin, Hindenburgdamm 30, 12203 Berlin, Germany

${ }^{\mathrm{b}}$ University of Oxford, Department of Experimental Psychology, Radcliffe Observatory, Anna Watts Building, Woodstock Rd, Oxford OX2 6GG, United Kingdom

${ }^{\mathrm{c}}$ University of Oxford, Department of Materials, OX2 6HT, Oxford, United Kingdom

Please address correspondence to: Roi Cohen Kadosh, roi.cohenkadosh@psy.ox.ac.uk

\begin{abstract}
While the treatment of Attention Deficit Hyperactivity Disorder (ADHD) is dominated by pharmacological agents, transcranial electrical stimulation (tES) is gaining attention as an alternative method for treatment. Most current meta-analyses have suggested that tES can improve cognitive functions that are otherwise impaired in ADHD, such as inhibition and working memory, as well as alleviated clinical symptoms. Here we review some of the promising findings in the field of tES. At the same time, we highlight two factors, which hinder the effective application of tES in treating ADHD: 1) the heterogeneity of tES protocols used in different studies; 2) patient profiles influencing responses to tES. We highlight potential solutions for overcoming such limitations, including the use of active machine learning, and provide simulated data to demonstrate how these solutions could also improve the understanding, diagnosis, and treatment of ADHD.
\end{abstract}

Keywords: tES, NIBS, ADHD, heterogeneity, personalization, Bayesian optimization

\section{Highlights}

- We review the effect of tES in the field of ADHD 
tES AND ADHD

- We highlight the heterogeneity of tES protocols

- We highlight the impact of patients profiles on tES efficacy

- We suggest personalizing tES using Bayesian optimization and provide simulated data

- We describe future directions to move the field forward

\section{Introduction}

Transcranial electrical stimulation (tES) comprises a group of non-invasive brain stimulation techniques, involving the passage of a weak electrical current (commonly up to 2 $\mathrm{mA}$ ) through one or more electrodes placed on the scalp. Stimulation can be delivered by applying direct current (tDCS), alternating current (tACS), or random noise (tRNS). Whilst in tDCS, current flows from one electrode (anode) to another (cathode), in tACS and tRNS current flows bi-directionally in an alternating manner (Reed and Cohen Kadosh, 2018). Through modulation of cortical excitability, tES techniques are believed to induce plastic changes and have been shown to augment a range of cognitive abilities in healthy participants (Fertonani and Miniussi, 2017; Polanía et al., 2018; Santarnecchi et al., 2015). This has sparked the application of tES in clinical populations in which cognition is impaired, including attention deficit hyperactivity disorder (ADHD).

ADHD is one of the most common neurodevelopmental disorders, characterized by core symptoms of inattention, hyperactivity, and impulsivity (Feldman and Reiff, 2014). When utilized in ADHD, tES (predominantly tDCS) has been associated with improvements in a number of clinical symptom measures (Allenby et al., 2018; Cachoeira et al., 2017; Soff et al., 2017), cognitive measures, including multiple aspects of memory and executive functioning (Bandeira et al., 2016; Breitling et al., 2016; Munz et al., 2015; Nejati et al., 2017; PrehnKristensen et al., 2014; Soltaninejad et al., 2015), as well as improvements of brain abnormalities associated with ADHD (Breitling et al., 2020; Dallmer-Zerbe et al., 2020; Sotnikova et al., 2017). While there are few studies showing no effect on behavior (Cosmo et al., 2015; Jacoby and Lavidor, 2018), potentially a result of publication bias, many have cited improvements in only some of the tested domains, with null effects on others. This has resulted in substantial variability across experimental results, with effect sizes ranging from very small to large (Salehinejad et al., 2020).

Even though tES may represent a promising alternative to stimulant medication (Rubio et al., 2016), clinical application is hindered by the diversity in findings (Salehinjad et al., 2019). 
tES AND ADHD

Here, we propose strategies for resolving heterogeneity in order to increase effect sizes in tES research on ADHD, which could also be adopted in treating other clinical populations.

Methodologically, the combinatorial explosion resulting from the many plausible sets of stimulation parameters may be combatted through a machine learning approach called Bayesian optimization (Shahriari et al., 2016). Clinically, patients presenting with differing profiles (either behaviorally or cortically) may profit from tailored interventions matching their specific deficits (Karalunas and Nigg, 2020).

\section{Targeting Variability in tES}

Despite being an established research tool, the ever-growing number of publications utilizing tES have revealed a large amount of variability in the efficacy of stimulation. Application of identical tES protocols has yielded large inter- and intra- individual variability, resulting in a lack of reliability across studies (Chew et al., 2015; Guerra et al., 2020; Krause and Cohen Kadosh, 2014). This is also true for tES results in ADHD, where large variability in performance and response to stimulation was hypothesized to have masked overall effects (Breitling et al., 2020, 2016). We would like to highlight two factors, which potentially contribute to such variability. One factor involves the heterogeneity of the protocols employed by studies; varying not only in the tES method chosen, but also in the choice of stimulation sites and the exact stimulation parameters used. A second factor stems from individual differences in responses to tES, such that optimal stimulation parameters may differ between individuals (Chew et al., 2015; Krause and Cohen Kadosh, 2014).

\section{Locality of stimulation}

A key component of administering tES is the selection of a location at which to stimulate. This decision is usually guided by previous neuroimaging and tES studies, which have linked a specific brain region with a behavior of interest. ADHD patients are often significantly impaired in executive functions, such as attention, working memory, cognitive flexibility, and perhaps most importantly; inhibitory control (Faraone et al., 2015). Inhibitory control describes the capacity to inhibit an ongoing or prepotent response in exchange for a more adaptive one. An influential theory of ADHD places inhibitory control at the heart of dysfunction, such that its primary deficiency leads to a subsequent breakdown of other executive functions, finally giving rise to ADHD symptoms (Barkley, 1997). Another influential theory (though less often targeted with tES) places greater emphasis on deficient signaling of delayed rewards. Due to this deficiency, rewards farther away in time seem less 


\section{tES AND ADHD}

profitable and are more often discounted, resulting in observable ADHD symptoms, such as the inability to efficiently work for prolonged periods of time (Sagvolden et al., 1998). The two theories have been placed in partially overlapping thalamo-cortico-striatal circuits (SonugaBarke, 2005), and components of this circuit have been targeted with tES.

One such component is the dorsolateral prefrontal cortex (DLPFC) (Sonuga-Barke, 2005), which is the prime target of the vast majority of tES-ADHD publications. Irrespective of stimulation amplitude, session number, and cathode placement, anodal tDCS studies over the left DLPFC were effective in alleviating clinical and behavioral measures of hyperactivity and impulsivity (Allenby et al., 2018; Soff et al., 2017), visual attention (Bandeira et al., 2016), working memory (Nejati et al., 2017), and connectivity in working memory networks (Sotnikova et al., 2017). However, the same stimulation only resulted in mixed effects on inhibitory control (Bandeira et al., 2016; Cosmo et al., 2015; Nejati et al., 2017; Soltaninejad et al., 2015). Other studies found that placing the cathode over the left DLPFC improved inhibitory control (Soltaninejad et al., 2015), while, anodal stimulation over the right DLPFC was shown to improve clinical measures of ADHD symptoms (Cachoeira et al., 2017) as well as inhibitory control (Nejati et al., 2017).

These results seem especially puzzling with respect to inhibitory control; with studies of anodal left, cathodal left, and anodal right DLPFC tDCS all reporting positive effects on inhibitory control. Proposed reasons include transcallosal connections between left and right DLPFC (Nejati et al., 2017; Soltaninejad et al., 2015), inhibitory control being a multicomponent concept including inference control and response inhibition (Breitling et al., 2016; Nejati et al., 2017), as well as different methods used to assess inhibitory control across studies (NEPSY-II, Go/No-Go, and Stroop Tasks) (Bandeira et al., 2016; Cosmo et al., 2015; Soltaninejad et al., 2015).

Another target of tES ADHD studies, which has also been implicated in inhibitory control and working memory (Clark et al., 2007), is the right inferior frontal gyrus (IFG). Applying anodal, cathodal, and sham tDCS over the right IFG in three separate sessions yielded no stimulation effect on inference control in an adolescents with ADHD (Breitling et al., 2016). In a similar sample, another study found that anodal stimulation over the right IFG by both high definition (HD-tDCS) and conventional tDCS increased P300 amplitudes to a level that resembled healthy controls. Though the P300 had previously been related to working memory, its increase did not translate into increased behavioral performance on a working memory task in this study (Breitling et al., 2020). 
tES AND ADHD

When right IFG and left DLPFC were simultaneously stimulated with tRNS, improvements in ADHD symptoms and working memory compared to anodal tDCS over the left DLPFC were observed (Berger et al., 2019). One potential conclusion drawn from this could be that tES over the right IFG does not lead to improvements of related behavior since two out of three studies found no effect and the third additionally stimulated the DLPFC, making it hard to disentangle the respective roles of these regions. However, the differential effect of stimulation in these studies could have possibly been influenced by other factors, including symptom profiles and current intensity. We will discuss both in the following sections.

A final target in tES studies was the orbitofrontal cortex (OFC), which is an area implicated in the reward processing theory of ADHD, as described in the beginning of this section (Sonuga-Barke, 2005). 1mA combined DLPFC-OFC tDCS significantly increased cognitive flexibility/task switching in children with ADHD, an effect that could not be established through DLPFC stimulation alone (Nejati et al., 2017).

A multi-electrode set up was utilized in one single study, where applying network level stimulation, aiming to target the bilateral temporal and parietal cortices, resulted in increases in P300 amplitude accompanied by a decrease of omission errors in adults with ADHD (DallmerZerbe et al., 2020). Only one pediatric study compared multiple electrode montages (Nejati et al., 2017) with very different results on performance on a battery of executive function tasks, depending on which area stimulated (Nejati et al., 2017). It is therefore likely that stimulation site, as well as matching of montage and task, have contributed to a significant proportion of variability across results.

\section{Stimulation parameters}

The results of tES have further been found to be amplitude, frequency, and (in the case of tACS) phase dependent (Alekseichuk et al., 2016; Harty and Cohen Kadosh, 2019; Santarnecchi et al., 2015). Even when stimulating the same target area and using the same task, different stimulation amplitudes may result in different effects. For example, two separate tDCS studies stimulated the left DLPFC for 15 minutes before assessing ADHD patients on a Go/NoGo Task (Nejati et al., 2017; Soltaninejad et al., 2015). No significant effect was found at $1 \mathrm{~mA}$ (Nejati et al., 2017), contrasting with $1.5 \mathrm{~mA}$ which resulted in an increased proportion of correct responses given in the "go stage" of the task (Soltaninejad et al., 2015). However, it should be noted that their designs differed in other factors, including cathode placement, participant age, and ADHD diagnostic assessment. Using 2mA tDCS, Allenby and colleagues 
tES AND ADHD

also found significant effects on behavior tapping into the same concept as the Go/No Go Task (the Stop-Signal Task) (Allenby et al., 2018). Thus, the effects of tDCS in ADHD depend on the current applied, which is an established finding in tES research overall (Batsikadze et al., 2013; Chew et al., 2015). However, the dose-response relationship between tDCS and behavior is poorly understood and may not be linear (Batsikadze et al., 2013). Similarly, the effects of tRNS on the performance of healthy participants has also been shown to vary as a function of the current applied (Harty and Cohen Kadosh, 2019). When applying sinusoidal current (as in tACS), the problem is further complicated as additional selection of frequency and phase parameters come into play. To achieve maximal increases in endogenous oscillations, the frequency of the applied current should match the frequency of the intrinsic oscillations (Schmidt et al., 2014) and should not be phase-shifted, as this would lead to cancellation instead of entrainment (Turi et al., 2020).

Finally, the use of a 'one-size-fits-all' approach is incongruous with what we know of the brain and how it functions. Optimal stimulation parameters may differ between individuals. For example, when healthy participants were exposed to four different tDCS current intensities $(0.2,0.5,1$, and $2 \mathrm{~mA})$, cluster analysis identified different clusters of participants that responded to the 0.2 and $2 \mathrm{~mA}$ (Chew et al., 2015). These results are not surprising considering the heterogeneity present in factors that may affect the outcome of tES, including but not limited to, participants' demographics, hormonal fluctuations, initial brain-state, and caffeine consumption, which could alter the relative excitation to relative inhibition, which has been assumed to influence the effect of brain stimulation (Krause et al., 2013; Sheffield et al., 2020). Accordingly, applying a single current dose to all participants will result in some receiving a more optimal amount and others receiving either excessive or insufficient amounts to achieve neuronal and behavioral effects. Similar findings have been reported with respect to frequency ranges. Numerous studies report variability between and within participants in the locations of specific peaks, such as alpha and theta along the frequency range (Benwell et al., 2019; Moran et al., 2010; Rodriguez-Larios and Alaerts, 2019). For example, when recording oscillatory activity during working memory retention and subsequently averaging over trials, participants with high and low working memory capacity showed different average peaks $(10.1 \mathrm{~Hz}$ and 12.4 $\mathrm{Hz}$ respectively), as well as different bandwidth around these peaks (wider for high capacity individuals) (Moran et al., 2010). Despite this, researchers often continue to apply stimulation at one specific frequency (e.g. Clayton et al., 2019) and either fail to take into account underlying individual differences or simply include them as covariates in post-hoc analyses. 
tES AND ADHD

\section{Machine learning solutions}

An issue researchers inevitably face when trying to solve the problem of identifying which stimulation parameters to use is the large number of combinations of parameters. For instance, if a researcher wished to stimulate using tACS they might have a range of currents from 0.1-2 $\mathrm{mA}$ and a range of frequencies from 1-50 Hz. Assuming a resolution of $0.1 \mathrm{~mA}$ and $1 \mathrm{~Hz}$, that is 1000 possible stimulation parameter combinations to choose from. To determine the ideal stimulation parameters through testing each combination individually would clearly be an enormous (and unrealistic) amount of work. One recent possible advance in the selection of stimulation parameters comes in the form of a machine learning algorithm called Bayesian optimization (Shahriari et al., 2016). Bayesian optimization is a method in which a parameter space, in the case of stimulation the current $x$ frequency space, may be efficiently searched to find the maximal outcome, such as a behavioral or neuronal response (Figure 2). Whilst a fixed search of each possible point would require a large number of samples, Bayesian optimization has the advantage of limiting the number of samples required whilst locating the maximal response (for a general review see Snoek et al. (2012), for a review in the field of cognitive neuroscience see Lorenz et al. (2017)). For ADHD, Bayesian optimization approaches could be used to find dose-response functions describing the relationship between stimulation parameters and behavioral outcome measures, such as, sustained attention, inhibitory control, or clinical symptoms. Alternatively, the algorithm could be utilized to find a latent function describing neuronal responses relevant to ADHD (like activity in the DLPFC, IFG, or electrophysiological changes), in relation to the specific parameters used. Finally, by taking into account baseline measures of cortical excitability, Bayesian optimization could further the understanding of which set of parameters is optimal for different individuals. This offers a much more data-driven approach to stimulation parameter selection, in comparison to the commonly used practice of reverting to the protocols previously used in the literature, without the empirical work to suggest the most suitable parameters over others. We provide an example of Bayesian optimization using simulated data in the last section of this review. 
tES AND ADHD
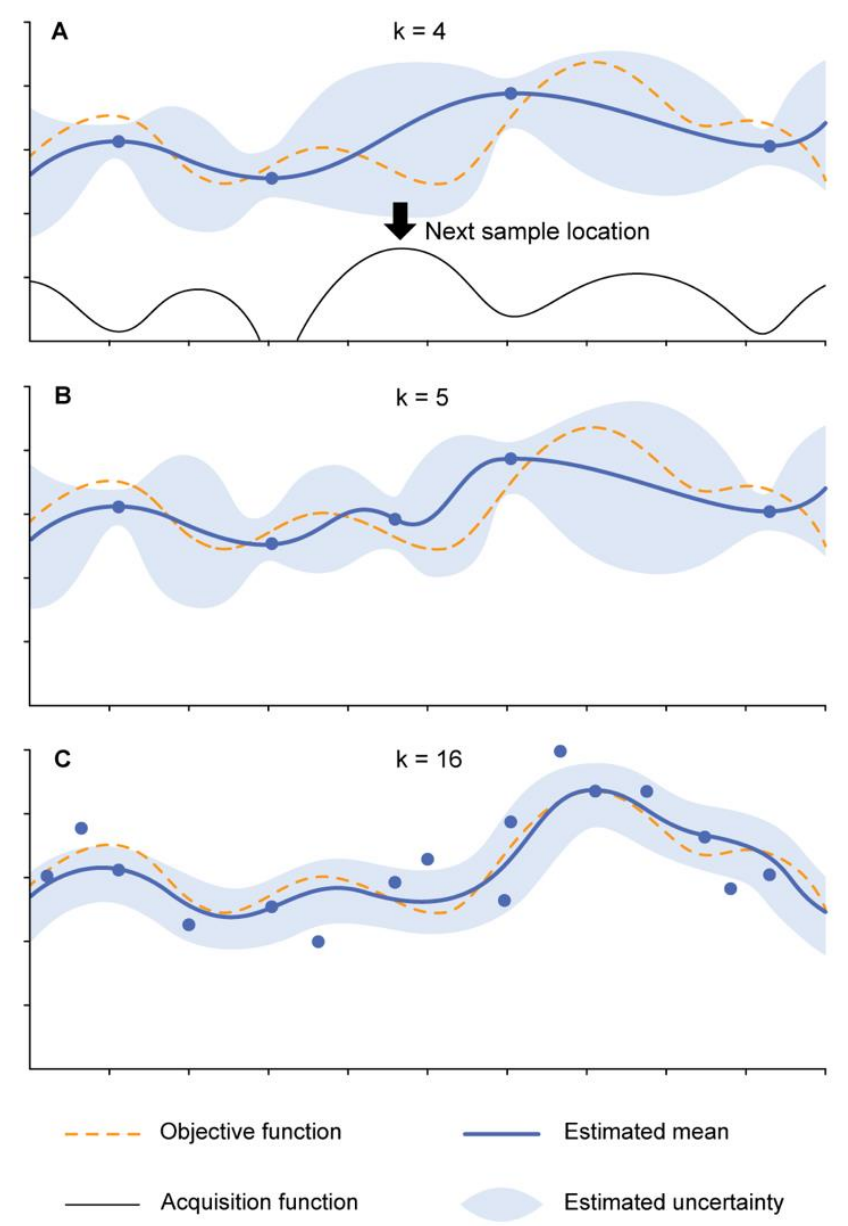

Figure 1. Illustration of Bayesian Optimization. The algorithm aims to approximate an unknown objective function, which links each parameter value ( $\mathrm{x}$ axis) to a behavioral/neuronal response (y axis). This is achieved in the following steps. A) Initial data points are sampled. A Gaussian process estimates a first mean function and its associated uncertainty. Then, an acquisition function locates the next parameter to sample at (e.g. where the uncertainty is large). B) The new data point is sampled and the Gaussian process is updated. C) After many iterations, the estimated mean function has approximated the objective function.

\section{Targeting Patient Heterogeneity}

In ADHD, variability in tES may be exacerbated through large within-syndrome heterogeneity. Not only does ADHD manifest differently depending whether an individual presents with the predominantly hyperactive/impulsive, predominantly inattentive, or combined subtype, even within subtypes there exists substantial variation on broader dimensional features (Nigg et al., 2020). Patients vary in levels of neurocognitive impairment: with some almost indistinguishable from typically developing individuals, and others with severe impairments in cognitive functions (Coghill et al., 2014; Lambek et al., 2018; Nigg et al., 2005). Another source of ADHD heterogeneity stems from differences in the temperament of patients: accumulating evidence points toward an emotionally dysregulated sub profile, which predicts clinical outcomes better than symptom severity or subtypes (Karalunas et al., 
tES AND ADHD

2019a; Nigg et al., 2020). Finally, ADHD subtypes, cognitive, and emotional impairments can change over the course of development and may even interact to produce symptoms (Nigg et al., 2020; Rabinovitz et al., 2016).

Despite knowledge of within syndrome heterogeneity, to date tES research on ADHD has not addressed this variation specifically. Even though most studies reported the ADHD subtypes of their participants, few have analyzed data separately for subtypes, or even applied symptom-specific protocols (for reviews see Rubio et al, 2016; Salehinejad et al., 2020). However, there is evidence that symptom profiles may influence stimulation efficacy. For example, when Breitling and her colleagues (2020) found no effect on working memory performance after $0.75 \mathrm{~mA}$ HD-tDCS over the right IFG in an adolescent sample, they speculated that the large between participant variability may have masked an overall effect. When subsequently dividing participants into 'responders' and 'non-responders', it was revealed that the response to HD-tDCS was inversely related to the number of hyperactive symptoms and that this effect was independent of baseline working memory performance. This led authors to suggest a tentative link between distinct neural characteristics of hyperactive and inattentive subtypes and the response to stimulation. Though a replication is needed to increase confidence in this finding, recording baseline symptom profiles may provide key insights to unravelling which patients respond to which type of stimulation.

\section{Symptom profiles}

As mentioned in the previous paragraph, Breitling and colleagues (2020) found the facilitatory effect of HD-tDCS on working memory performance to be dependent on hyperactive symptoms. Other studies also found selective effects of stimulation on some symptoms only. In another adolescent sample, Soff and colleagues (2017) found $1 \mathrm{~mA}$ left anodal DLPFC tDCS to selectively improve behavioral measures of inattention and hyperactivity, but not impulsivity. The discrepancy in these symptom effects can be resolved by consulting their concurrent fMRI analysis. tDCS not only increased activation and connectivity in the DLPFC and its related working memory network, but also in neighboring motor networks (Sotnikova et al., 2017). Thus, neuroplasticity-related effects in working memory networks (including the DLPFC) may have helped to ameliorate inattentiveness, while spill-over effects to motor networks may have reduced hyperactivity. The moderate effects on impulsivity were justified by the fact that this montage did not target areas related to inhibitory control, such as the IFG or medial PFC (Soff et al., 2017). Although further research with more focal stimulation protocols should rigorously test these hypotheses, this opens a potentially 
tES AND ADHD

exciting new avenue for personalizing montages to specific patient groups. While predominantly hyperactive patients may profit most from motor network stimulation, predominantly inattentive subtypes may profit more from working memory network stimulation, and combined subtypes from combined stimulation.

\section{Cognitive profiles}

Next to core symptoms, broader dimensions of cognitive and emotional functioning contribute to heterogeneity in ADHD and may influence stimulation efficacy. While the evidence argues quite clearly for an existence of distinct cognitive profiles, there is still disagreement concerning the nature of these profiles (Karalunas and Nigg, 2020; Nigg et al., 2020). Some argue for individual differences with specific strength and weakness profiles (quantitative), while others believe in a non-specific severity pattern with some individuals generally doing worse than others (qualitative) (Karalunas and Nigg, 2020). With respect to quantitative subgroups, evidence is most consistent for subgroups characterized by executive functioning deficits (including working memory and inhibition), and response time deficits (slow and/or variable) (Karalunas and Nigg, 2020). All of these deficits have been targeted in tES ADHD studies, yielding very mixed results. While a number of studies reported increases in working memory performance following tDCS over the left DLPFC (Berger et al., 2019; Nejati et al., 2017; Soff et al., 2017), others have not (Bandeira et al., 2016). Results are similarly mixed for inhibitory control, with some indications of tDCS over the left DLPFC increasing inhibitory control (Bandeira et al., 2016; Munz et al., 2015; Nejati et al., 2017; Soltaninejad et al., 2015), and others finding no effect (Allenby et al., 2018; Cosmo et al., 2015). Finally, the same holds for response time and response time variability: some found improvements following tES (Breitling et al., 2016; Munz et al., 2015; Soff et al., 2017) and others did not (Allenby et al., 2018; Dallmer-Zerbe et al., 2020). Admittedly, these findings are hard to compare and a large portion of variability is probably attributable to differences in design, as the studies varied in tES method, session numbers, stimulation duration and parameters, as well as electrode number and placement. However, one factor that may have additionally contributed to variability in findings concerns the cognitive profiles of participants. Since the studies were all conducted in very small samples of ADHD patients, different distributions of cognitive profiles may have skewed results accordingly. Patients who are actually impaired a cognitive function of interest may profit more from tES than those who are not. For example, the largest effect of working memory training with concurrent $1 \mathrm{~mA}$ tDCS over the left DLPFC was attained for those healthy adults with the lowest baseline working 
tES AND ADHD

memory performance (Ruf et al., 2017). Systematically investigating cognitive profiles with tES may synergistically provide novel insights into both lines of work. If individually targeting cognitive profiles reduces between subject variability in tES results, this would allow for better predictions of who will respond to stimulation and potentially inform future personalization approaches based on cognitive dimensions. At the same time, such findings could validate the existence of the identified cognitive subgroups and advance the understanding of ADHD heterogeneity.

Another important consideration concerns the relationship between cognitive profiles and ADHD symptoms. From a clinical standpoint, tES-related improvements in cognitive profiles would have to be accompanied by improvements in hallmark symptoms and functional outcomes of patients, in order to render it relevant for treatment. As mentioned previously (see introduction, locality of stimulation, and symptom profile sections), studies investigating changes in ADHD symptoms following tES report positive results in children (Berger et al., 2019), adolescents (Soff et al., 2017), as well as adults (Allenby et al., 2018; Cachoeira et al., 2017). One study additionally reported improvements in self-reported functional outcomes (work, school, family life) of adult ADHD patients following five sessions of $2 \mathrm{~mA}$ left anodal DLPFC tDCS (Cachoeira et al., 2017). Whether these clinical effects are driven by improvements in cognitive functioning is currently unclear. Some studies have assessed symptoms and functioning via clinical questionnaires only, without simultaneously considering cognitive functions (Cachoeira et al., 2017). Others have investigated behavioral measures of ADHD symptoms via attention and working memory tasks (Allenby et al., 2018; Soff et al., 2017), thus equating clinical improvements with cognitive improvements. Finally, studies assessing both clinical questionnaires as well as cognitive performance also yielded equivocal results. For example, in the $1 \mathrm{~mA}$ left anodal DLPFC tDCS paradigm of Soff and colleagues (2017), stimulation resulted in conflicting effects on clinical and behavioral measures of ADHD symptoms. While tDCS induced improvements in inattention and impulsivity on parent-rated questionnaires, behavioral measures (QbTest) indicated reductions in inattention and hyperactivity (Soff et al., 2017). Only one study specifically investigated interactions between clinical and cognitive changes (Berger et al., 2019). When comparing tDCS and tRNS, greater tRNS-related improvements on clinical symptom questionnaires were predicted by greater behavioral WM improvements from baseline (Berger et al., 2019). Future studies should therefore aim to replicate these initial findings and include both clinical measures of symptoms and functioning, as well as cognitive performance measures, in order to clarify the role of cognition to the observed clinical improvements following tES. 
tES AND ADHD

\section{Emotional profiles}

With respect to the emotional dimension, growing evidence suggests reproducible subgroups along temperament features (Karalunas and Nigg, 2020; Nigg et al., 2020). Multiple clustering approaches yielded similar profiles across the age range; one exhibiting normative emotional functioning, one extraverted/sensation seeking group, and one group characterized by negative affect/neuroticism (Karalunas et al., 2019b; Karalunas and Nigg, 2020; Smith and Martel, 2019). Because both extremes of the temperament spectrum predicted worsening of clinical outcomes, some authors suggested that a broader distinction between ADHD with and without emotional dysregulation may be more informative than a distinction between valence of dysregulation (Karalunas and Nigg, 2020). Despite growing recognition of emotional subtyping in ADHD, the literature on neural correlates is sparse. Some evidence suggests distinct EEG frontal alpha power profiles for adolescent ADHD with high and low negative affect (Alperin et al., 2019). Others have found whole brain elevations of alpha and beta power to be related to internalizing behaviors and emotion dysregulation in both ADHD and healthy children (Loo et al., 2018). With the field in its infancy, no tES study has yet included emotional profiles as variables, or even stimulated its putative neural correlates. However, with future replications of aberrant EEG oscillations in emotional dysfunction, emotional dimensions may be targeted with tACS to examine the casual role of EEG oscillations in emotional dysregulation in ADHD. Eventually, taking into account emotional profiles may also aid future personalization approaches and help elucidate heterogeneity of tES results.

\section{Developmental stage}

Finally, ADHD subtypes as well as cognitive and emotional profiles are not stable and may change over the lifespan. While preschoolers often present with motor restlessness and disruptive or aggressive behaviors, adolescents and adults more often present with inattentive, impulsive, and disorganized symptoms (Luo et al., 2019). Further, with the asynchronous maturation of limbic and prefrontal structures during adolescence, specific cognitive and emotional profiles may emerge during this period (Nigg et al., 2020). Taking into account developmental stage may thus provide further insights into the specific ADHD profile of patients and thereby aid personalization of tES. Furthermore, emotional profiles have been found to interact with cognition over development. This is illustrated by one study following children from the age three to seven, showing that early emotional dysregulation (negative affect and anger dysregulation) drove later ADHD symptom severity via disruption of executive functioning (Rabinovitz et al., 2016). It has been proposed that these interactions may result in 
tES AND ADHD

a vicious cycle of emotionality and disruptions of top down control, mutually influencing each other (Nigg et al., 2020). Intervening at an early stage could potentially break the cycle and prevent serious cognitive dysfunctions, which eventually amount to ADHD symptoms hindering educational and occupational success (Fredriksen et al., 2014). As mentioned in the previous paragraph, some evidence suggests that emotional profiles are characterized by distinct EEG signatures (Alperin et al., 2019; Loo et al., 2018), offering potential targets for early tES interventions.

\section{Targeting Brain Profiles}

Instead of characterizing disorders based on symptom lists, which often do not align with findings from clinical neuroscience, the National Institute of Mental Health sought alternative approaches to classification and started the Research Domain Criteria (RDoC) project. Rather than going inward from observable symptoms, RDoC focusses on neurobiology and aims to link aberrant circuitry to behavior. While maintaining a focus on psychopathology, disorders are viewed as dimensional, and they may overlap due to transdiagnostic risk factors (Insel et al., 2010). In the previous section, it was described how going from the outside in, tES could be customized to the specific symptom protocol of participants through adapted protocols. A personalization approach more in line with $\mathrm{RDoC}$ would be to bypass diagnostic 
tES AND ADHD

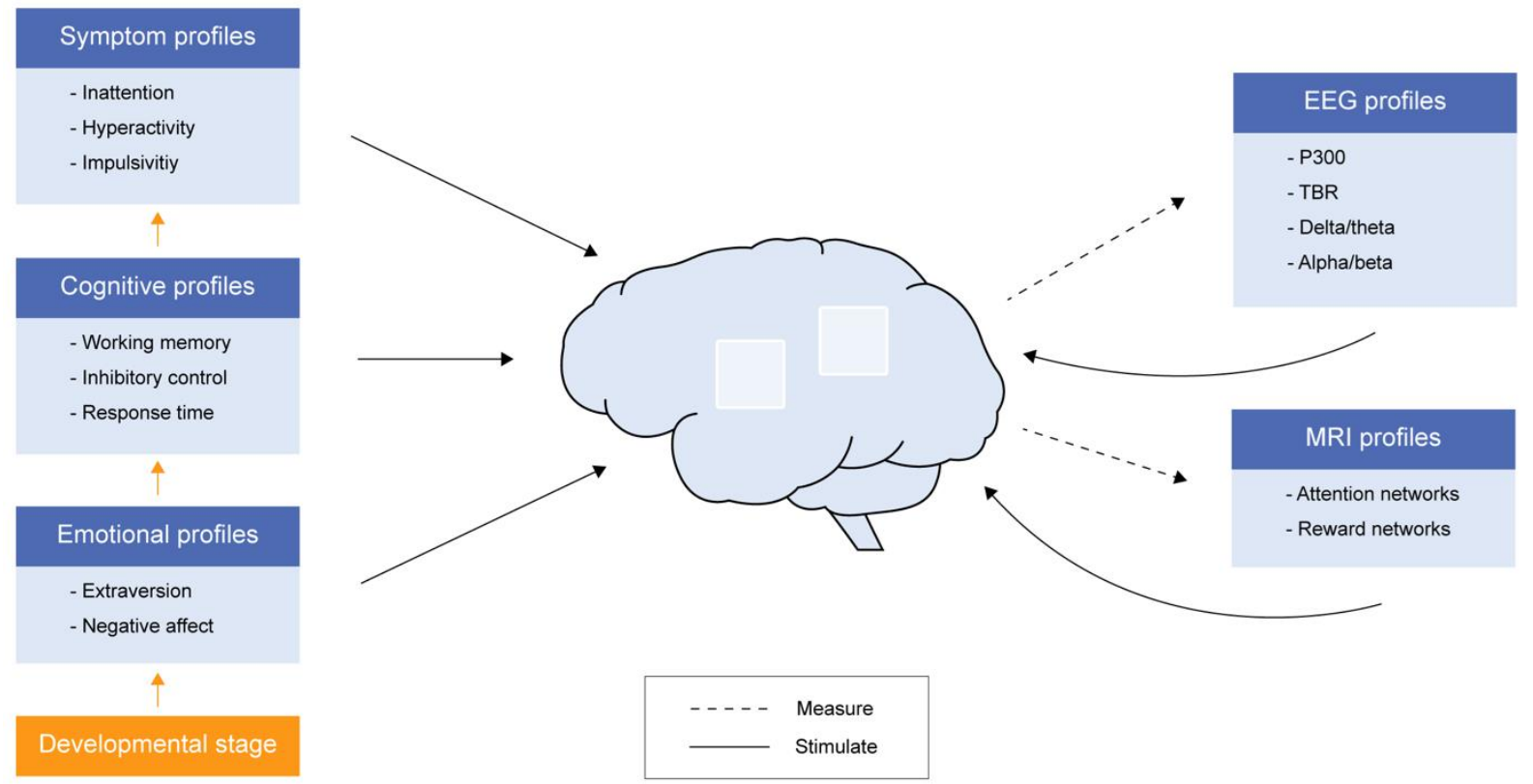

Figure 2. Avenues for targeting ADHD heterogeneity with tES. Left: going from observable ADHD sub profiles to identifying neural targets for adapted stimulation (group level). Right: going from latent brain profiles to individually adapting stimulation, in order to observe effects on ADHD symptoms (individual level). Boxes overlaying the brain represent just two of the many possible stimulation locations. Note that this illustration does not represent an exhaustive list, but is rather exemplary for possible future tES interventions.

$\mathrm{ADHD}=$ attention deficit hyperactivity disorder, $\mathrm{EEG}=$ electroencephalography, $\mathrm{MRI}=$ magnetic resonance imaging, P300 = EEG component observable during visual oddball tasks, TBR $=$ theta/beta ratio, $\mathrm{tES}=$ transcranial electrical stimulation

interpretation and immediately apply tES to aberrant brain activity in order to observe its effects on behavior (see Figure 2 for a summary of both approaches).

A recent study presents a first glance at what personalized methods for ADHD could look like. Using an elegant tACS protocol, Dallmer-Zerbe and her colleagues (2020) successfully increased the $\mathrm{P} 300$ amplitude of ADHD patients by taking into account individual differences. The P300 is an EEG component observable in delta and theta ranges when discriminating targets from standards (e.g. in visual oddball tasks). It has been related to working memory and its amplitude is reportedly decreased in ADHD patients, as compared to healthy controls. Next to amplitude, people also vary in P300 latency, and this variation was specifically targeted in their design. After performing an initial visual oddball task without stimulation, participant data was briefly preprocessed to estimate each individual's P300 latency. This estimated latency was then specifically targeted through applying tACS at a frequency and phase matched to the individual. The resulting increases in P300 amplitudes were accompanied by a behavioral reduction of omission errors (Dallmer-Zerbe et al., 2020). Customizing tES through frequency estimation, phase-locked to endogenous patterns, may be 
tES AND ADHD

an especially interesting avenue for further research, since multiple abnormalities in EEG frequency spectra have been reported for ADHD.

\section{EEG profiles}

Perhaps the most famous EEG biomarker suggested for ADHD concerns an elevation of theta- relative to beta power bands (theta/beta ratio, TBR) over frontocentral electrodes (Arns et al., 2013). A number of early studies have found large effect sizes when attempting to classify whether an individual had ADHD or not on the basis of TBR (Lubar, 1991; Monastra et al., 1999). Replications eventually amounted to the food and drug administration approving of TBR as a diagnostic marker for ADHD (Saad et al., 2018). However, more recently the clinical utility of this method has been questioned heavily due to inconsistent findings (Arns et al., 2013; Saad et al., 2018). Meta-analytic results suggest that there is a substantial subgroup of ADHD patients exhibiting elevated TBR, and that, within this subgroup, TBR is a favorable predictor of response to stimulant medication and neurofeedback interventions (Arns et al., 2013). Although other characteristics of this subgroup were not specified, baseline TBR assessments could aid personalization of tES to only targeting those participants who actually deviate on this measure. Similarly to what was described for the P300, theta and beta bands could be targeted through phase-locked tACS in the respective frequency bands.

Moving away from TBR measures over frontocentral electrodes, heterogeneity in whole brain EEG patterns have also been investigated in a large sample of children with and without ADHD (Loo et al., 2018). Results revealed five separable EEG profiles, each (except one) with a different elevation in one of the pre-defined frequency bands. The profiles were behaviorally meaningful, such that participants with elevations in slow-wave activity (delta, theta) exhibited higher levels of cognitive dysfunction and externalizing behaviors, and elevations in higher frequency bands (alpha, beta) showed intact cognitive functions but greater levels of internalizing behaviors and emotion dysregulation (Loo et al., 2018). This corroborates well with the cognitive and emotional sub profiles proposed for ADHD (Karalunas and Nigg, 2020; Nigg et al., 2020). However, it is important to note that the distribution of healthy and ADHD participants was similar across subgroups, suggesting that there is no EEG profile for children with and without ADHD. Rather, brain based profiles seem to code for susceptibility to specific cognitive or emotional styles, which may more generally heighten the risk of psychopathology. These transdiagnostic factors are again in line with RDoC approaches and offer tES targets for cognitive and emotional profiles in ADHD. Baseline EEG assessments may help identify those patients who could benefit the most from stimulation in the respective frequency ranges. 
tES AND ADHD

\section{MRI profiles}

The small number of studies adopting neurobiological subtyping approaches using MRI have focused on connectivity between pre-defined regions of interest, rather than adopting truly data driven approaches (Costa Dias et al., 2015; Gates et al., 2014; Lecei et al., 2019). For example, one study adopted a machine learning approach which aimed to arrive at connectivity subgroups within ADHD and healthy controls based on pre-defined regions within the frontoparietal network. Similarly to what was found in EEG subtyping approaches, MRI results also showed that none of the resulting subgroups were entirely filled with ADHD or healthy probands. Out of the five groups, two were predominantly filled with ADHD patients and three predominantly filled with healthy controls. The two ADHD dominated groups exhibited different connectivity patterns, one with weaker DLPFC-intraparietal sulcus connectivity, and one with heightened connectivity between these areas. The latter group also exhibited decreased connectivity between left and right DLPFC (Gates et al., 2014). A similar approach was taken by another study, this time investigating connectivity between reward-system areas (Costa Dias et al., 2015). Three subgroups could be identified, each with differing patterns of connectivity between the nucleus accumbens and a number of areas relevant to adaptive decision making. All subgroups were again comprised of both healthy and ADHD participants. Within one of the subgroups, ADHD patients were significantly more impulsive than both the healthy controls within their group, as well as the ADHD patients of other groups.

Though MRI based subtyping holds promise for future personalization approaches based on individual connectivity patterns, the evidence to date is too sparse to arrive at meaningful translations for tES. With relatively small sample sizes and pre-defined regions of interest, reproducibility and interpretation of these findings have yet to be established.

\section{Simulated Data}

We designed a simulated function to demonstrate the potential utility of taking into account ADHD heterogeneity in order to tailor optimal tES parameters using personalized Bayesian optimization (van Bueren et al., 2020). We set up the function with two input dimensions: (1) tES Current Intensity (mA) ranging from 0.1 to 2.0 and (2) Neurophysiology (TBR) ranging from 0.6 to 3.0. The Neurophysiology was considered as the personalized variable, which is specific to each participant. The output of our simulated function was the clinical outcome, which for illustrative purposes ranged from -1 (poor outcome) to 1 (desired outcome). 

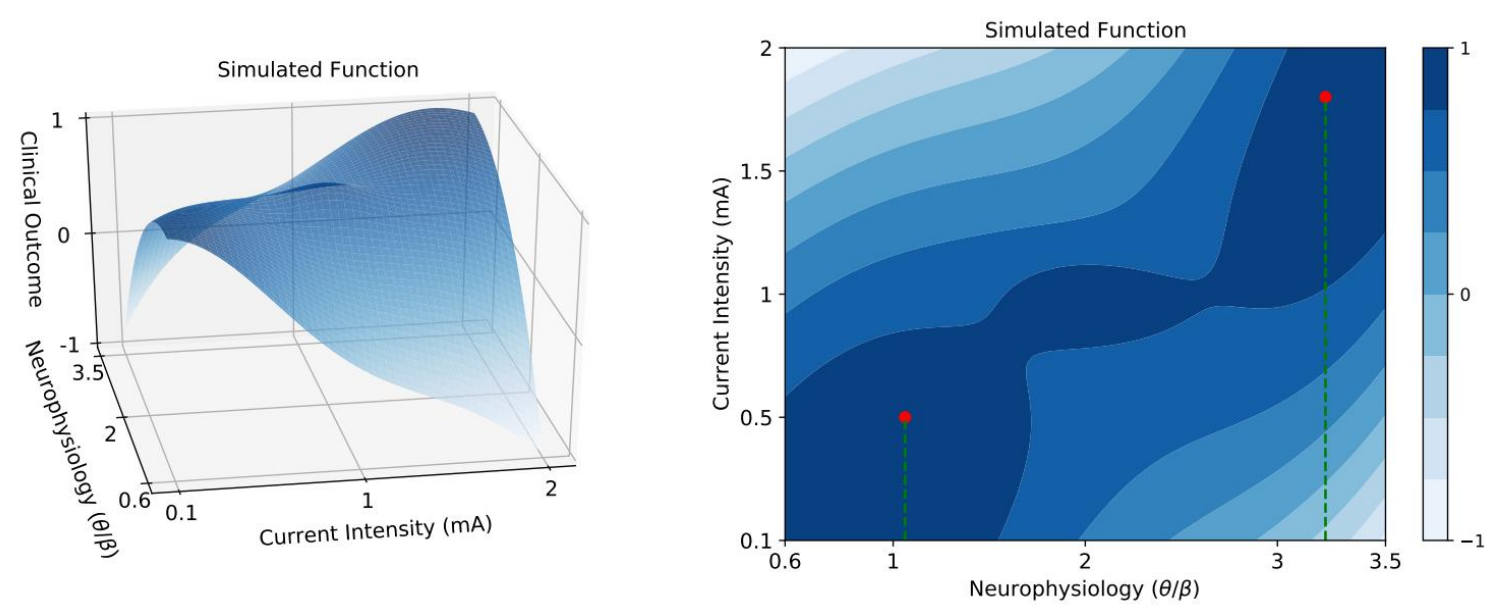

Figure 3 Examples of our simulated function in 2D view (right) and 3D view (left). Given the personalized variable Neurophysiology, we selected the Current Intensity, tested the function, and observed the Clinical Outcome.

We visualized our function in Figure 3. Based on previous findings, we expected those with higher TBR to require a higher current than those with lower TBR (Harty and Cohen Kadosh, 2019). Given the personalized variable of Neurophysiology, we selected the Current Intensity, tested the function, and observed the Clinical Outcome. Note that it is neither possible to control nor optimize this personalized variable.

\section{Algorithm training}

We ran and compared three approaches: 1) Random search, 2) non-personalized Bayesian optimization (hereafter, Bayesian optimization), and 3) personalized Bayesian optimization, over 30 iterations including 6 randomly chosen points at the beginning. The program was implemented in Python. Each iteration took two seconds to suggest a new parameter. The implementation for Bayesian optimization can be found at https://github.com/ntienvu/KnownOptimum_BO (Nguyen and Osborne, 2020).

The true optimal parameter for each value of Neurophysiology, which is unknown to the scientist/clinician, is shown as black dots in Figure 4. We can see that different Neurophysiology values will require different Current Intensity to allow for the best Clinical Outcome. 
tES AND ADHD

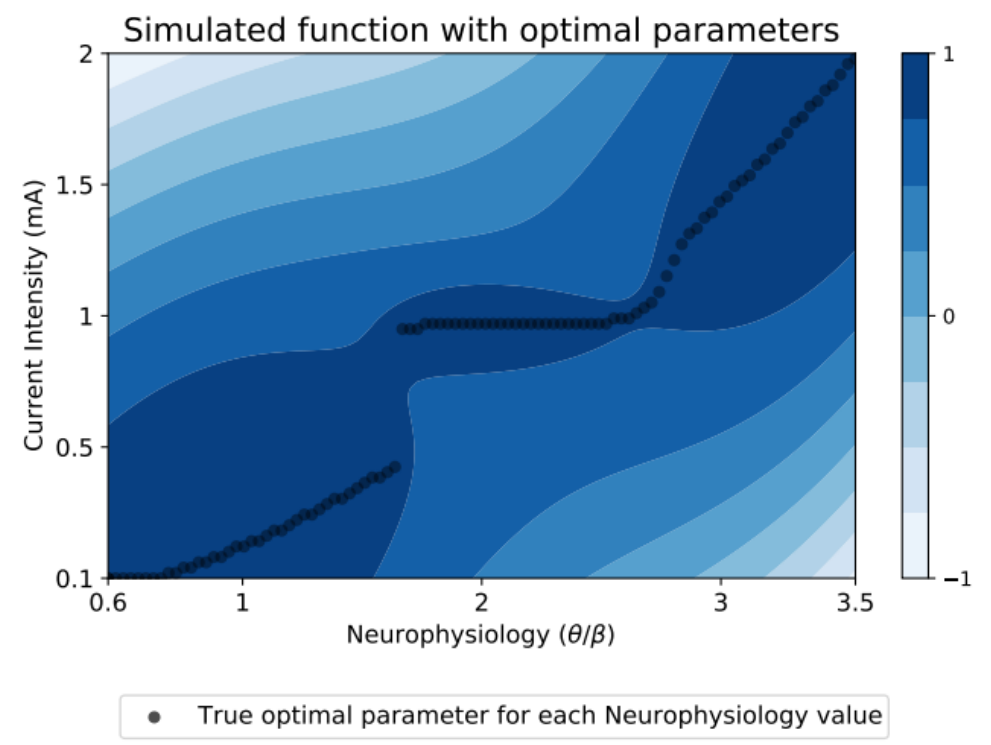

Figure 4. The simulated function and true optimal parameters (black dots) for each Neurophysiology value. Note that these true optimal parameters are unknown to the scientist/clinician.

We visualized the best parameter estimated at the final iteration by Bayesian optimization and personalized Bayesian optimization in Figure 5. Particularly, the suggested parameters by personalized Bayesian optimization (green dots) are very close to the true optimal parameters (black dots). In contrast, Bayesian optimization, which does not consider the personalized Neurophysiology values, suggests a flat line (in red).

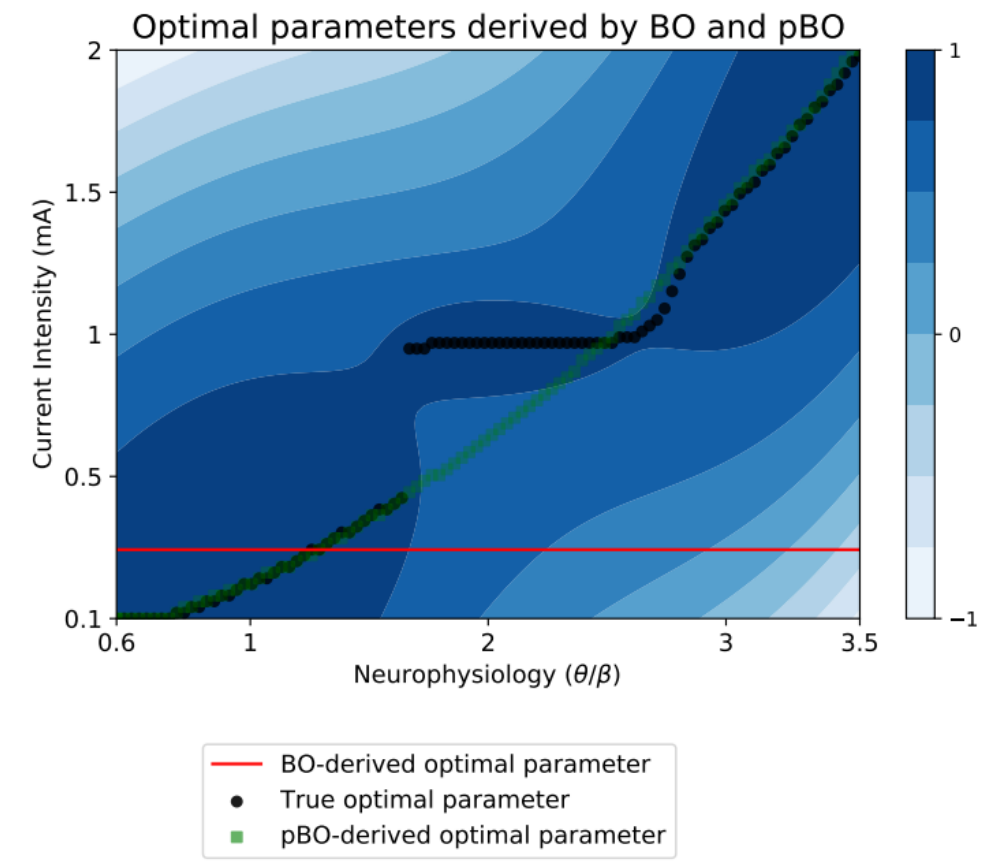

Figure 5. Overlaying the suggested parameters of each algorithm. Given the Neurophysiology value, personalized Bayesian optimization (pBO) will suggest the parameters (green dot) close to the true optimal ones (black dots). Bayesian optimization (BO) does not consider the Neurophysiology value. 
Next, we plot the selected parameters by personalized Bayesian optimization over iterations in Figure 6. The randomly chosen points from the initial phase are displayed in magenta and the points selected by personalized Bayesian optimization are shown in green. These points are conditioned on the Neurophysiology value, as indicated by the green dashed lines. From this it becomes evident that personalized Bayesian optimization can identify the high-performing region, as most of its selected parameters remain within the high value region (dark blue color).One desirable property of personalized Bayesian optimization is that, while mainly selecting points in high value regions, it preserves some probability for exploration to gain information about the underlying function.

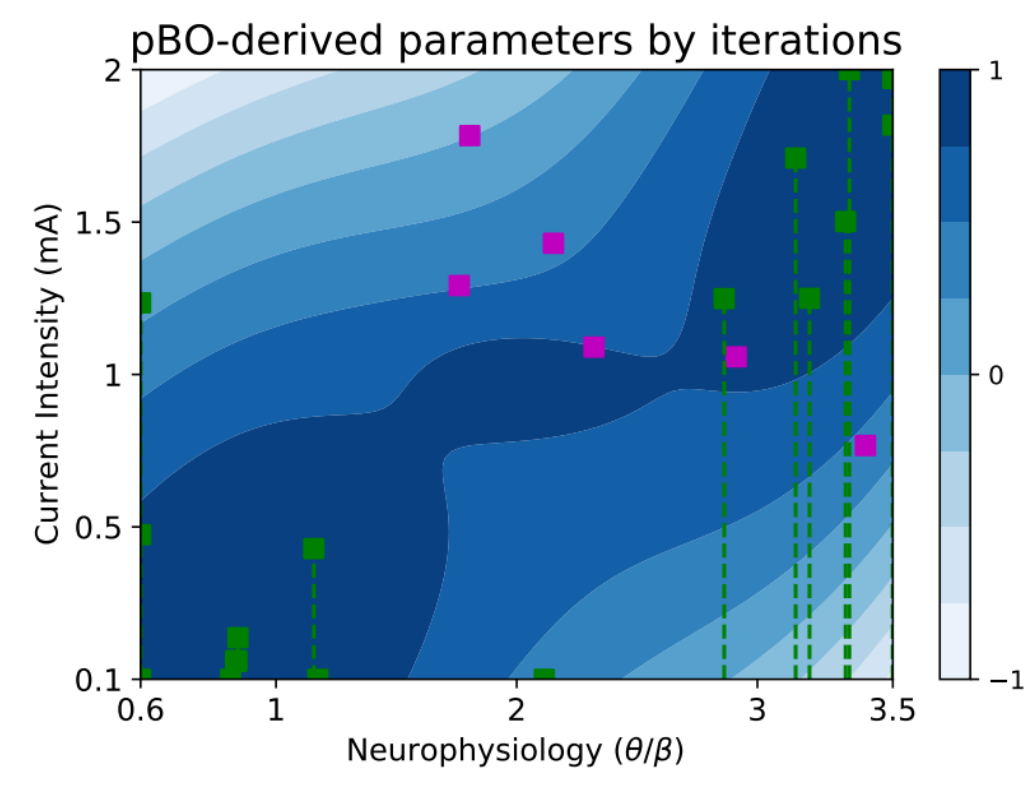

- Random Phase a Current Intensity selected

Figure 6. Parameters selected by personalized Bayesian optimization (pBO) over iterations. These parameters are conditioned on the Neurophysiology values.

Finally, we compared the performance across methods in Figure 7, which shows that personalized Bayesian optimization outperformed both Bayesian optimization and Random Search in terms of providing the best clinical outcome. It is interesting to note that the performance of personalized Bayesian optimization at an early stage (at iteration 10) is achieved only later by Bayesian optimization and Random Search (after 20 and 30 iterations, respectively). In other words, personalized Bayesian optimization is two times faster than 
tES AND ADHD

Bayesian optimization and three times faster than Random Search, therefore requiring significantly less resources.

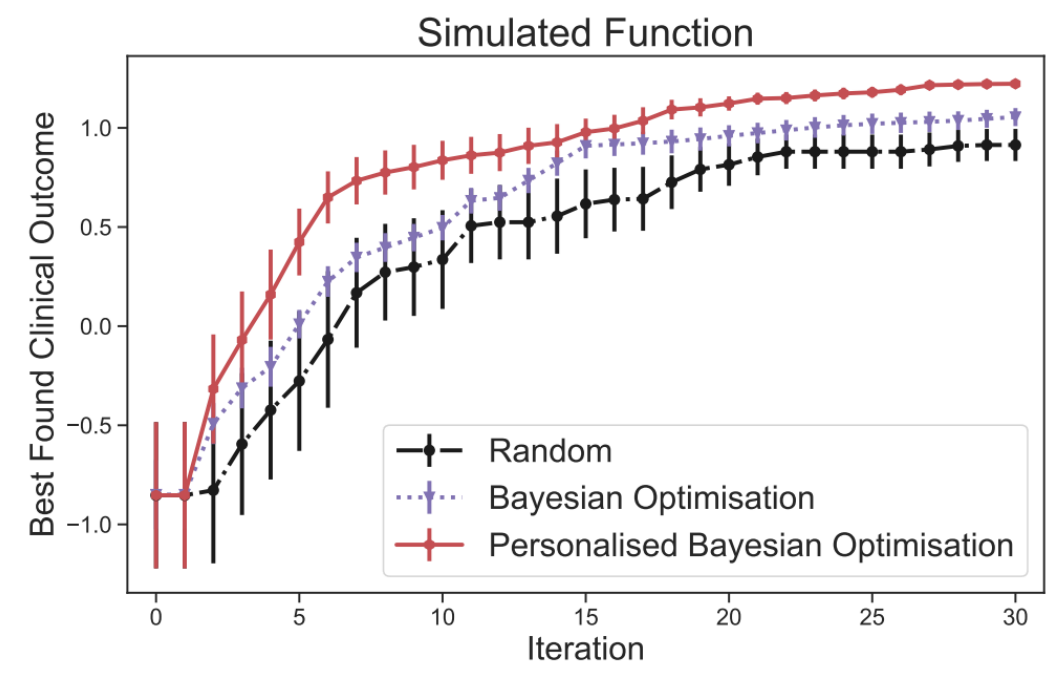

Figure 7. Comparison between Random search, Bayesian optimization and personalized Bayesian optimization.

\section{Conclusions and Future Directions}

tES research on ADHD has yielded variable, yet promising results. These findings include alleviating clinical symptoms and improving cognitive function despite potentially heterogeneous data. Here we discussed two main sources of variability: heterogeneity of stimulation protocols and heterogeneity of the patient group and suggest how future studies could reduce the sources of inter- and intra-experimental variability. Firstly, stimulation efficacy varies with differing stimulation sites and parameters employed by studies. Because testing each possible combination of stimulation parameters could quickly escalate into an intractable amount of work, we proposed that optimal combinations may be found using machine learning. Bayesian optimization algorithms could efficiently search the parameter space in order to find latent functions relating a specific set of parameters to relevant predictor or outcome variables. We extended such data-driven approach to personalized Bayesian optimization and demonstrated, using simulated data, how it could identify functions relating each level of baseline neurophysiology, in this case TBR, to tailor the most suitable stimulation parameters. Other resulting functions could describe the relationship between stimulation parameters and attentional and inhibitory performance, or activity in related networks. In this way, learning about individual stimulation optima represents a novel way of endorsing long standing knowledge of variability in tES and may lead to more adaptive future stimulation approaches. 
tES AND ADHD

Another direction is to target multiple regions in order to have more impact at the network level, rather than targeting a single region (Breitling et al., 2020). While a proof-of-concept assessed the feasibility of such approach in ADHD, further studies will allow providing more conclusive evidence (Karstens and Cohen Kadosh, 2020).

The second source of variability in results presumably stems from large withinsyndrome heterogeneity. Patients vary not only in subtype but also degree of cognitive and emotional impairment. Despite knowledge of ADHD heterogeneity, few tES studies to date have addressed this variation. We therefore proposed how personalized tES approaches may help resolve variable results. First evidence suggests that ADHD subtype may influence stimulation success and that different tES protocols may alleviate different types of symptoms. Personalizing montages to the specific symptom profile of patients may thus increase overall efficacy. Cognitive impairments of ADHD have also been targeted with tES in a global manner, without addressing baseline variability of the patient group - again with largely variable results. Personalizing tES to specific cognitive profiles could therefore increase stimulation efficacy, as well as validate previously proposed cognitive profiles, thereby advancing future ADHD subtyping approaches. When investigating cognitive subtyping of ADHD using tES, studies should further aim to include clinical severity measures, in order to carefully scrutinize whether tES-related changes in cognitive performance are accompanied by or mediate clinically relevant changes in symptoms or functional outcomes. Emotionally dysregulated ADHD profiles have thus far not been targeted with tES. However, since emotional profiles have been related to distinct EEG signatures, they may also contribute to heterogeneity of tES results and offer targets for personalized interventions. This may be an intriguing avenue for future research since early emotional dysregulation has been identified as a possible starting point to a vicious cycle of emotional dysregulation and cognitive impairment mutually driving each other.

Moreover, instead of going from observable symptoms to possible stimulation targets, one could also start at the other end and directly target aberrant neural patterns of ADHD. Approaches like these could add valuable data to the $\mathrm{RDoC}$ project and help move away from traditional diagnostic, which currently is based on self-report, toward a neurobiological objective measure. However, findings of brain profiles of ADHD are again heterogeneous and there is no consensus as to which profiles are meaningful. Instead of working to resolve this heterogeneity, we proposed that it could be specifically targeted. Initial evidence suggests that estimating parameters on a single-participant basis can achieve group level cortical and behavioral effects. Future tES applications could therefore be customized to the specific EEG or MRI profile of patients. In this respect, the inclusion of neuroimaging could further reduce 
tES AND ADHD

variability via using functional MRI to adjust electrode position based on individual brain activation, which have been suggested to allow greater accuracy in stimulation and as a result increased effect size (Sack et al., 2009).

Finally, the move from single session tES experiments toward multiple sessions tES is warrant. Many studies are using single-session tES designs and the motivation for doing that is multiple, including a reduced burden on the experimenter and the patient, an easier and faster completion of the experiment and sending work for publication, as well as a significantly reduced budget requested from funding bodies. However, the translation of such results to therapy is rather weak and our view is that future studies should move towards multiple sessions tES and expanding its impact beyond the cognitive level to clinical symptoms. Previous studies have suggested that repeated tES sessions increase the strength of the tES effect and the likelihood of long-lasting effect (Brevet-Aeby et al., 2019; Krause and Cohen Kadosh, 2013). A single session application might be too weak to result in any apparent behavioral change, while it may lead to a temporary change at the neural level (Clemens et al., 2013; Mosbacher et al., 2020; Snowball et al., 2013) that with further sessions could be translated to the behavioral level.

Overall, we know of many variables that are likely to influence the effectiveness of tES on an individual basis. Accordingly, ADHD as a heterogeneous patient group is unlikely to profit from a fixed protocol of tES. If we acknowledge heterogeneity in both tES and ADHD, we could increase effect sizes through machine learning and personalized interventions. Eventually, this may allow a stronger testbed for the existence of different pathophysiology associated with subtypes of ADHD and result in effective tES protocols that could be applied successfully in clinical practice.

\section{Declaration of Interest}

RCK serves on the scientific advisory boards for Neuroelectrics and Innosphere. In addition, RCK TLR and VN filed a UK Patent via the University of Oxford for "method for obtaining personalized parameters for transcranial stimulation, transcranial system, method of applying transcranial stimulation". This patent is based on Bayesian optimization we describe in this manuscript. MB serves on the scientific advisory board of GH research and Johnson \& Johnson. EA serves on the national advisory board Shire/Takeda. No other authors indicate conflict of interest. 
tES AND ADHD

\section{Acknowledgements}

OxBer Seed Funding II (Reference Number: OXBERII_CH1). The funders had no role in the study design or data collection. 
tES AND ADHD

\section{References}

Alekseichuk, I., Turi, Z., Amador de Lara, G., Antal, A., Paulus, W., 2016. Spatial Working Memory in Humans Depends on Theta and High Gamma Synchronization in the Prefrontal Cortex. Current Biology 26, 1513-1521. https://doi.org/10.1016/j.cub.2016.04.035

Allenby, C., Falcone, M., Bernardo, L., Wileyto, E.P., Rostain, A., Ramsay, J.R., Lerman, C., Loughead, J., 2018. Transcranial direct current brain stimulation decreases impulsivity in ADHD. Brain Stimulation 11, 974-981. https://doi.org/10.1016/j.brs.2018.04.016

Alperin, B.R., Smith, C.J., Gustafsson, H.C., Figuracion, M.T., Karalunas, S.L., 2019. The relationship between alpha asymmetry and ADHD depends on negative affect level and parenting practices. Journal of Psychiatric Research 116, 138-146. https://doi.org/10.1016/j.jpsychires.2019.06.016

Arns, M., Conners, C.K., Kraemer, H.C., 2013. A Decade of EEG Theta/Beta Ratio Research in ADHD: A Meta-Analysis. J Atten Disord 17, 374-383. https://doi.org/10.1177/1087054712460087

Bandeira, I.D., Guimarães, R.S.Q., Jagersbacher, J.G., Barretto, T.L., de Jesus-Silva, J.R., Santos, S.N., Argollo, N., Lucena, R., 2016. Transcranial Direct Current Stimulation in Children and Adolescents With Attention-Deficit/Hyperactivity Disorder (ADHD): A Pilot Study. J Child Neurol 31, 918-924. https://doi.org/10.1177/0883073816630083

Barkley, R.A., 1997. Behavioral Inhibition, Sustained Attention, and Executive Functions: Constructing a Unifying Theory of ADHD 30. https://doi.org/10.1037/00332909.121.1.65

Batsikadze, G., Moliadze, V., Paulus, W., Kuo, M.-F., Nitsche, M.A., 2013. Partially non-linear stimulation intensity-dependent effects of direct current stimulation on motor cortex excitability in humans: Effect of tDCS on cortical excitability. The Journal of Physiology 591, 1987-2000. https://doi.org/10.1113/jphysiol.2012.249730

Benwell, C.S.Y., London, R.E., Tagliabue, C.F., Veniero, D., Gross, J., Keitel, C., Thut, G., 2019. Frequency and power of human alpha oscillations drift systematically with timeon-task. NeuroImage 192, 101-114. https://doi.org/10.1016/j.neuroimage.2019.02.067

Berger, I., Dakwar-Kawar, O., Grossman, E.S., Nahum, M., Cohen Kadosh, R., 2019. Scaffolding the Attention-Deficit/Hyperactivity Disorder Brain Using Random Noise Stimulation (preprint). Psychiatry and Clinical Psychology. https://doi.org/10.1101/19005983

Breitling, C., Zaehle, T., Dannhauer, M., Bonath, B., Tegelbeckers, J., Flechtner, H.-H., Krauel, 
tES AND ADHD

K., 2016. Improving Interference Control in ADHD Patients with Transcranial Direct $\begin{array}{llllll}\text { Current Stimulation } & \text { (tDCS). Front. Cell. Neurosci. } & 10 .\end{array}$ https://doi.org/10.3389/fncel.2016.00072

Breitling, C., Zaehle, T., Dannhauer, M., Tegelbeckers, J., Flechtner, H.-H., Krauel, K., 2020. Comparison between conventional and HD-tDCS of the right inferior frontal gyrus in children and adolescents with ADHD. Clinical Neurophysiology 131, 1146-1154. https://doi.org/10.1016/j.clinph.2019.12.412

Brevet-Aeby, C., Mondino, M., Poulet, E., Brunelin, J., 2019. Three repeated sessions of transcranial random noise stimulation (tRNS) leads to long-term effects on reaction time in the Go/No Go task. Neurophysiologie Clinique 49, 27-32. https://doi.org/10.1016/j.neucli.2018.10.066

Cachoeira, C.T., Leffa, D.T., Mittelstadt, S.D., Mendes, L.S.T., Brunoni, A.R., Pinto, J.V., Blazius, V., Machado, V., Bau, C.H.D., Rohde, L.A., Grevet, E.H., Schestatsky, P., 2017. Positive effects of transcranial direct current stimulation in adult patients with attention-deficit/hyperactivity disorder A pilot randomized controlled study. Psychiatry Research 247, 28-32. https://doi.org/10.1016/j.psychres.2016.11.009

Chew, T., Ho, K.-A., Loo, C.K., 2015. Inter- and Intra-individual Variability in Response to Transcranial Direct Current Stimulation (tDCS) at Varying Current Intensities. Brain Stimulation 8, 1130-1137. https://doi.org/10.1016/j.brs.2015.07.031

Clark, L., Blackwell, A.D., Aron, A.R., Turner, D.C., Dowson, J., Robbins, T.W., Sahakian, B.J., 2007. Association Between Response Inhibition and Working Memory in Adult ADHD: A Link to Right Frontal Cortex Pathology? Biological Psychiatry 61, 13951401. https://doi.org/10.1016/j.biopsych.2006.07.020

Clayton, M.S., Yeung, N., Cohen Kadosh, R., 2019. Electrical stimulation of alpha oscillations stabilizes performance on visual attention tasks. Journal of Experimental Psychology: General 148, 203-220. https://doi.org/10.1037/xge0000502

Clemens, B., Jung, S., Zvyagintsev, M., Domahs, F., Willmes, K., 2013. Modulating arithmetic fact retrieval: A single-blind, sham-controlled tDCS study with repeated fMRI $\begin{array}{lll}\text { measurements. } & \text { Neuropsychologia } & \text { 1279-1286. }\end{array}$ https://doi.org/10.1016/j.neuropsychologia.2013.03.023

Coghill, D.R., Seth, S., Matthews, K., 2014. A comprehensive assessment of memory, delay aversion, timing, inhibition, decision making and variability in attention deficit hyperactivity disorder: advancing beyond the three-pathway models. Psychol. Med. 44, 1989-2001. https://doi.org/10.1017/S0033291713002547 
tES AND ADHD

Cosmo, C., Baptista, A.F., de Araújo, A.N., do Rosário, R.S., Miranda, J.G.V., Montoya, P., de Sena, E.P., 2015. A Randomized, Double-Blind, Sham-Controlled Trial of Transcranial Direct Current Stimulation in Attention-Deficit/Hyperactivity Disorder. PLoS ONE 10, e0135371. https://doi.org/10.1371/journal.pone.0135371

Costa Dias, T.G., Iyer, S.P., Carpenter, S.D., Cary, R.P., Wilson, V.B., Mitchell, S.H., Nigg, J.T., Fair, D.A., 2015. Characterizing heterogeneity in children with and without ADHD based on reward system connectivity. Developmental Cognitive Neuroscience 11, 155174. https://doi.org/10.1016/j.dcn.2014.12.005

Dallmer-Zerbe, I., Popp, F., Lam, A.P., Philipsen, A., Herrmann, C.S., 2020. Transcranial Alternating Current Stimulation (tACS) as a Tool to Modulate P300 Amplitude in Attention Deficit Hyperactivity Disorder (ADHD): Preliminary Findings. Brain Topogr 33, 191-207. https://doi.org/10.1007/s10548-020-00752-X

Faraone, S.V., Asherson, P., Banaschewski, T., Biederman, J., Buitelaar, J.K., Ramos-Quiroga, J.A., Rohde, L.A., Sonuga-Barke, E.J.S., Tannock, R., Franke, B., 2015. Attentiondeficit/hyperactivity disorder. Nat Rev Dis Primers 1, 15020. https://doi.org/10.1038/nrdp.2015.20

Feldman, H.M., Reiff, M.I., 2014. Attention Deficit-Hyperactivity Disorder in Children and Adolescents. N Engl J Med 370, 838-846. https://doi.org/10.1056/NEJMcp1307215

Fertonani, A., Miniussi, C., 2017. Transcranial Electrical Stimulation: What We Know and Do Not Know About Mechanisms. Neuroscientist 23, 109-123. https://doi.org/10.1177/1073858416631966

Fredriksen, M., Dahl, A.A., Martinsen, E.W., Klungsoyr, O., Faraone, S.V., Peleikis, D.E., 2014. Childhood and persistent ADHD symptoms associated with educational failure and long-term occupational disability in adult ADHD. ADHD Atten Def Hyp Disord 6, 87-99. https://doi.org/10.1007/s12402-014-0126-1

Gates, K.M., Molenaar, P.C.M., Iyer, S.P., Nigg, J.T., Fair, D.A., 2014. Organizing Heterogeneous Samples Using Community Detection of GIMME-Derived Resting State Functional Networks. PLoS ONE 9, e91322. https://doi.org/10.1371/journal.pone.0091322

Guerra, A., López-Alonso, V., Cheeran, B., Suppa, A., 2020. Variability in non-invasive brain stimulation studies: Reasons and results. Neuroscience Letters 719, 133330. https://doi.org/10.1016/j.neulet.2017.12.058

Harty, S., Cohen Kadosh, R., 2019. Suboptimal Engagement of High-Level Cortical Regions Predicts Random-Noise-Related Gains in Sustained Attention. Psychol Sci 30, 1318- 
tES AND ADHD

1332. https://doi.org/10.1177/0956797619856658

Insel, T., Cuthbert, B., Garvey, M., Heinssen, R., Pine, D.S., Quinn, K., Sanislow, C., Wang, P., 2010. Research Domain Criteria (RDoC): Toward a New Classification Framework for Research on Mental Disorders. AJP 167, 748-751. https://doi.org/10.1176/appi.ajp.2010.09091379

Jacoby, N., Lavidor, M., 2018. Null tDCS Effects in a Sustained Attention Task: The Modulating Role of Learning. Front. Psychol. 9, 476. https://doi.org/10.3389/fpsyg.2018.00476

Karalunas, S.L., Gustafsson, H.C., Fair, D., Musser, E.D., Nigg, J.T., 2019a. Do we need an irritable subtype of ADHD? Replication and extension of a promising temperament profile approach to ADHD subtyping. Psychological Assessment 31, 236-247. https://doi.org/10.1037/pas0000664

Karalunas, S.L., Gustafsson, H.C., Fair, D., Musser, E.D., Nigg, J.T., 2019b. Do we need an irritable subtype of ADHD? Replication and extension of a promising temperament profile approach to ADHD subtyping. Psychological Assessment 31, 236-247. https://doi.org/10.1037/pas0000664

Karalunas, S.L., Nigg, J.T., 2020. Heterogeneity and Subtyping in AttentionDeficit/Hyperactivity Disorder - Considerations for Emerging Research Using PersonCentered Computational Approaches. Biological Psychiatry 88, 103-110. https://doi.org/10.1016/j.biopsych.2019.11.002

Karstens, M.I., Cohen Kadosh, R., 2020. Targeting neuronal correlates of executive function in ADHD using brain stimulation. Clinical Neurophysiology 131, 1142-1143. https://doi.org/10.1016/j.clinph.2020.01.015

Krause, B., Cohen Kadosh, R., 2014. Not all brains are created equal: the relevance of individual differences in responsiveness to transcranial electrical stimulation. Front. Syst. Neurosci. 8. https://doi.org/10.3389/fnsys.2014.00025

Krause, B., Cohen Kadosh, R., 2013. Can transcranial electrical stimulation improve learning difficulties in atypical brain development? A future possibility for cognitive training. Developmental Cognitive Neuroscience 6, 176-194. https://doi.org/10.1016/j.den.2013.04.001

Krause, B., Márquez-Ruiz, J., Kadosh, R.C., 2013. The effect of transcranial direct current stimulation: a role for cortical excitation/inhibition balance? Front. Hum. Neurosci. 7. https://doi.org/10.3389/fnhum.2013.00602

Lambek, R., Sonuga-Barke, E., Tannock, R., Sørensen, A.V., Damm, D., Thomsen, P.H., 2018. 
tES AND ADHD

Are there distinct cognitive and motivational sub-groups of children with ADHD? Psychol. Med. 48, 1722-1730. https://doi.org/10.1017/S0033291717003245

Lecei, A., van Hulst, B.M., de Zeeuw, P., van der Pluijm, M., Rijks, Y., Durston, S., 2019. Can we use neuroimaging data to differentiate between subgroups of children with ADHD symptoms: A proof of concept study using latent class analysis of brain activity. NeuroImage: Clinical 21, 101601. https://doi.org/10.1016/j.nicl.2018.11.011

Loo, S.K., McGough, J.J., McCracken, J.T., Smalley, S.L., 2018. Parsing heterogeneity in attention-deficit hyperactivity disorder using EEG -based subgroups. J Child Psychol Psychiatr 59, 223-231. https://doi.org/10.1111/jcpp.12814

Lorenz, R., Hampshire, A., Leech, R., 2017. Neuroadaptive Bayesian Optimization and Hypothesis Testing. Trends in Cognitive Sciences 21, 155-167. https://doi.org/10.1016/j.tics.2017.01.006

Lubar, J.F., 1991. Discourse on the development of EEG diagnostics and biofeedback for attention-deficit/hyperactivity disorders. Biofeedback and Self-Regulation 16, 201-225. https://doi.org/10.1007/BF01000016

Luo, Y., Weibman, D., Halperin, J.M., Li, X., 2019. A Review of Heterogeneity in Attention Deficit/Hyperactivity Disorder (ADHD). Front. Hum. Neurosci. 13, 42. https://doi.org/10.3389/fnhum.2019.00042

Monastra, V.J., Lubar, J.F., Linden, M., VanDeusen, P., Green, G., Wing, W., Phillips, A., Fenger, T.N., 1999. Assessing attention deficit hyperactivity disorder via quantitative electroencephalography: An initial validation study. Neuropsychology 13, 424-433. https://doi.org/10.1037/0894-4105.13.3.424

Moran, R.J., Campo, P., Maestu, F., Reilly, R.B., Dolan, R.J., Strange, B.A., 2010. Peak Frequency in the Theta and Alpha Bands Correlates with Human Working Memory Capacity. Front. Hum. Neurosci. 4. https://doi.org/10.3389/fnhum.2010.00200

Mosbacher, J.A., Brunner, C., Nitsche, M.A., Grabner, R.H., 2020. Effects of Anodal tDCS on Arithmetic Performance and Electrophysiological Activity. Front. Hum. Neurosci. 14, 17. https://doi.org/10.3389/fnhum.2020.00017

Munz, M.T., Prehn-Kristensen, A., Thielking, F., Mölle, M., Göder, R., Baving, L., 2015. Slow oscillating transcranial direct current stimulation during non-rapid eye movement sleep improves behavioral inhibition in attention-deficit/hyperactivity disorder. Front. Cell. Neurosci. 9. https://doi.org/10.3389/fncel.2015.00307

Nejati, V., Salehinejad, M.A., Nitsche, M.A., Najian, A., Javadi, A.-H., 2017. Transcranial Direct Current Stimulation Improves Executive Dysfunctions in ADHD: Implications 
tES AND ADHD

for Inhibitory Control, Interference Control, Working Memory, and Cognitive $\begin{array}{llll}\text { Flexibility. } & \text { J } & \text { Atten }\end{array}$ https://doi.org/10.1177/1087054717730611

Nguyen, V., Osborne, M.A., 2020. Knowing The What But Not The Where in Bayesian Optimization. In International Conference on Machine Learning (pp. 7317-7326). PMLR.

Nigg, J.T., Karalunas, S.L., Feczko, E., Fair, D.A., 2020. Toward a Revised Nosology for Attention-Deficit/Hyperactivity Disorder Heterogeneity. Biological Psychiatry: Cognitive Neuroscience and Neuroimaging S2451902220300483. https://doi.org/10.1016/j.bpsc.2020.02.005

Nigg, J.T., Willcutt, E.G., Doyle, A.E., Sonuga-Barke, E.J.S., 2005. Causal Heterogeneity in Attention-Deficit/Hyperactivity Disorder: Do We Need Neuropsychologically Impaired Subtypes? $\quad$ Biological $\quad$ Psychiatry $\quad 57, \quad 1224-1230$. https://doi.org/10.1016/j.biopsych.2004.08.025

Polanía, R., Nitsche, M.A., Ruff, C.C., 2018. Studying and modifying brain function with noninvasive brain stimulation. Nat Neurosci 21, 174-187. https://doi.org/10.1038/s41593017-0054-4

Prehn-Kristensen, A., Munz, M., Göder, R., Wilhelm, I., Korr, K., Vahl, W., Wiesner, C.D., Baving, L., 2014. Transcranial Oscillatory Direct Current Stimulation During Sleep Improves Declarative Memory Consolidation in Children With Attentiondeficit/hyperactivity Disorder to a Level Comparable to Healthy Controls. Brain Stimulation 7, 793-799. https://doi.org/10.1016/j.brs.2014.07.036

Rabinovitz, B.B., O’Neill, S., Rajendran, K., Halperin, J.M., 2016. Temperament, executive control, and attention-deficit/hyperactivity disorder across early development. Journal of Abnormal Psychology 125, 196-206. https://doi.org/10.1037/abn0000093

Reed, T., Cohen Kadosh, R., 2018. Transcranial electrical stimulation (tES) mechanisms and its effects on cortical excitability and connectivity. J Inherit Metab Dis 41, 1123-1130. https://doi.org/10.1007/s10545-018-0181-4

Rodriguez-Larios, J., Alaerts, K., 2019. Tracking Transient Changes in the Neural Frequency Architecture: Harmonic Relationships between Theta and Alpha Peaks Facilitate Cognitive Performance. J. Neurosci. 39, 6291-6298. https://doi.org/10.1523/JNEUROSCI.2919-18.2019

Rubio, B., Boes, A.D., Laganiere, S., Rotenberg, A., Jeurissen, D., Pascual-Leone, A., 2016. Noninvasive Brain Stimulation in Pediatric Attention-Deficit Hyperactivity Disorder 
tES AND ADHD

(ADHD): A Review. J Child Neurol 31, 784-796. https://doi.org/10.1177/0883073815615672

Ruf, S.P., Fallgatter, A.J., Plewnia, C., 2017. Augmentation of working memory training by transcranial direct current stimulation (tDCS). Sci Rep 7, 876. https://doi.org/10.1038/s41598-017-01055-1

Saad, J.F., Kohn, M.R., Clarke, S., Lagopoulos, J., Hermens, D.F., 2018. Is the Theta/Beta EEG Marker for ADHD Inherently Flawed? J Atten Disord 22, 815-826. https://doi.org/10.1177/1087054715578270

Sack, A.T., Cohen Kadosh, R., Schuhmann, T., Moerel, M., Walsh, V., Goebel, R., 2009. Optimizing Functional Accuracy of TMS in Cognitive Studies: A Comparison of Methods. Journal of Cognitive Neuroscience 21, 207-221. https://doi.org/10.1162/jocn.2009.21126

Sagvolden, T., Aase, H., Zeiner, P., Berger, D., 1998. Altered reinforcement mechanisms in attention-deficit/hyperactivity disorder. Behavioural Brain Research 94, 61-71. https://doi.org/10.1016/S0166-4328(97)00170-8

Salehinejad, M.A., Nejati, V., Mosayebi-Samani, M., Mohammadi, A., Wischnewski, M., Kuo, M.-F., Avenanti, A., Vicario, C.M., Nitsche, M.A., 2020. Transcranial Direct Current Stimulation in ADHD: A Systematic Review of Efficacy, Safety, and Protocol-induced Electrical Field Modeling Results. Neurosci. Bull. https://doi.org/10.1007/s12264-02000501-x

Santarnecchi, E., Brem, A.-K., Levenbaum, E., Thompson, T., Kadosh, R.C., Pascual-Leone, A., 2015. Enhancing cognition using transcranial electrical stimulation. Current Opinion in Behavioral Sciences 4, 171-178. https://doi.org/10.1016/j.cobeha.2015.06.003

Schmidt, S.L., Iyengar, A.K., Foulser, A.A., Boyle, M.R., Fröhlich, F., 2014. Endogenous Cortical Oscillations Constrain Neuromodulation by Weak Electric Fields. Brain Stimulation 7, 878-889. https://doi.org/10.1016/j.brs.2014.07.033

Shahriari, B., Swersky, K., Wang, Z., Adams, R.P., de Freitas, N., 2016. Taking the Human Out of the Loop: A Review of Bayesian Optimization. Proc. IEEE 104, 148-175. https://doi.org/10.1109/JPROC.2015.2494218

Sheffield, J.G., Raz, G., Sella, F., Kadosh, R.C., 2020. How can noise alter neurophysiology in order to improve human behaviour? A combined tRNS and EEG study (preprint). Neuroscience. https://doi.org/10.1101/2020.01.09.900118

Smith, T.E., Martel, M.M., 2019. Trait-Based Profiles of ADHD in Adolescents and Young Adults. Journal of Clinical Child \& Adolescent Psychology 48, 440-454. 
tES AND ADHD

https://doi.org/10.1080/15374416.2018.1491004

Snoek, J., Larochelle, H., Adams, R.P., 2012. Practical Bayesian Optimization of Machine Learning Algorithms 9.

Snowball, A., Tachtsidis, I., Popescu, T., Thompson, J., Delazer, M., Zamarian, L., Zhu, T., Cohen Kadosh, R., 2013. Long-Term Enhancement of Brain Function and Cognition Using Cognitive Training and Brain Stimulation. Current Biology 23, 987-992. https://doi.org/10.1016/j.cub.2013.04.045

Soff, C., Sotnikova, A., Christiansen, H., Becker, K., Siniatchkin, M., 2017. Transcranial direct current stimulation improves clinical symptoms in adolescents with attention deficit hyperactivity disorder. J Neural Transm 124, 133-144. https://doi.org/10.1007/s00702016-1646-y

Soltaninejad, Z., Nejati, V., Ekhtiari, H., 2015. Effect of Anodal and Cathodal Transcranial Direct Current Stimulation on DLPFC on Modulation of Inhibitory Control in ADHD. J Atten Disord 23, 325-332. https://doi.org/10.1177/1087054715618792

Sonuga-Barke, E.J.S., 2005. Causal Models of Attention-Deficit/Hyperactivity Disorder: From Common Simple Deficits to Multiple Developmental Pathways. Biological Psychiatry 57, 1231-1238. https://doi.org/10.1016/j.biopsych.2004.09.008

Sotnikova, A., Soff, C., Tagliazucchi, E., Becker, K., Siniatchkin, M., 2017. Transcranial Direct Current Stimulation Modulates Neuronal Networks in Attention Deficit Hyperactivity Disorder. Brain Topogr 30, 656-672. https://doi.org/10.1007/s10548-017-0552-4

Turi, Z., Mittner, M., Lehr, A., Bürger, H., Antal, A., Paulus, W., 2020. $\theta-\gamma$ Cross-Frequency Transcranial Alternating Current Stimulation over the Trough Impairs Cognitive Control. eNeuro 7, ENEURO.0126-20.2020. https://doi.org/10.1523/ENEURO.0126$\underline{20.2020}$

van Bueren, N.E.R., Reed, T.L., Nguyen, V., Sheffield, J.G., van der Ven, S.H.G., Osborne, M.A., Kroesbergen, E.H., Cohen Kadosh, R. 2020. Personalized Closed-Loop Brain Stimulation for Effective Neurointervention. Manuscript submitted for publication. 